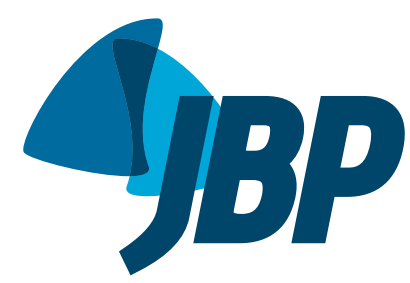

1. Disciplina de Cirurgia Torácica, Faculdade de Medicina, Universidade de São Paulo, São Paulo (SP) Brasil.

2. Hospital Israelita Albert Einstein, São Paulo (SP) Brasil.

3. Disciplina de Tórax, Escola Paulista de Medicina, Universidade Federal de São Paulo, São Paulo (SP) Brasil.

4. Centro Paulista de Oncologia, São Paulo (SP) Brasil.

Submitted: 23 July 2015

Accepted: 9 May 2016

Study carried out at the Hospital Israelita Albert Einstein and at the Faculdade de

Medicina, Universidade de São Paulo, São Paulo (SP) Brasil.

\section{Applications for a hybrid operating room in thoracic surgery: from multidisciplinary procedures to image-guided video-assisted thoracoscopic surgery}

Ricardo Mingarini Terra ${ }^{1,2}$, Juliano Ribeiro Andrade ${ }^{2}$, Alessandro Wasum Mariani1 ${ }^{1,2}$, Rodrigo Gobbo Garcia², Jose Ernesto Succi ${ }^{2,3}$, Andrey Soares ${ }^{2,4}$, Paulo Marcelo Zimmer ${ }^{2}$

\begin{abstract}
The concept of a hybrid operating room represents the union of a high-complexity surgical apparatus with state-of-the-art radiological tools (ultrasound, CT, fluoroscopy, or magnetic resonance imaging), in order to perform highly effective, minimally invasive procedures. Although the use of a hybrid operating room is well established in specialties such as neurosurgery and cardiovascular surgery, it has rarely been explored in thoracic surgery. Our objective was to discuss the possible applications of this technology in thoracic surgery, through the reporting of three cases.
\end{abstract}

Keywords: Thoracic surgery, video-assisted; Bronchoscopy; Thoracoscopy; Radiology, interventional

\section{INTRODUCTION}

A hybrid operating room (HOR) can be defined as a high-complexity operating room, fully equipped for minimally invasive procedures, such as video-assisted and robotic-assisted procedures, and provided with imaging tools, such as angiography, ultrasound, CT, or magnetic resonance imaging. (1) Such tools allow intraoperative image acquisition, assisting in planning and performing minimally invasive procedures. In this context, the integration of imaging and surgery is maximized, greatly increasing the accuracy of these procedures. ${ }^{(2)}$ In some specialties, such as neurosurgery ${ }^{(3)}$ cardiac surgery, ${ }^{(4)}$ and vascular surgery, ${ }^{(5)}$ the importance of an HOR is well established, allowing various compound procedures, such as percutaneous angioplasty plus myocardial revascularization during the same anesthesia. However, the role of an HOR in other specialties has yet to be defined.

Thoracic surgery is a specialty that can also benefit from the use of an HOR. The increasing use of video-assisted thoracoscopic surgery and robotic-assisted surgery associated with the frequent diagnosis of semisolid lesions, which are difficult to palpate/visualize intraoperatively, is a context in which the integration of imaging methods in the operating room can be very useful. Although few series have reported the use of an HOR in such circumstances, their results are promising.

The objective of the present article was to illustrate, through the reporting of three cases, some of the potential applications for an HOR in thoracic surgery: the performance of multiple diagnostic and therapeutic procedures during the same surgical session; and the intraoperative localization of nodules, in addition to determination of resection margins.

\section{CASE REPORTS}

\section{Case 1}

A 73-year-old male smoker presented with a mass in the right upper lobe associated with mediastinal lymph node enlargement. The radiological profile was consistent with primary lung cancer; however, during endoscopic aspiration biopsy of the subcarinal lymph node, uncertainty arose as to whether it was a granulatomous lesion. Therefore, we opted for performing a transthoracic biopsy, as well as a mediastinal lymph node biopsy by mediastinoscopy, and, depending on the results of the intraoperative analysis, we would also insert an indwelling central catheter for chemotherapy. The patient was brought to the HOR, and the procedures were performed sequentially. Elective endotracheal intubation, large-bore peripheral venous access, and an indwelling urinary catheter were used. The patient was initially placed in the right lateral decubitus position for performance of a percutaneous Tru-Cut needle biopsy, with the gantry (Artis Zeego; Siemens, Munich, Germany) set to tomographic mode, by the interventional radiology team. Pathological examination of the biopsy specimen revealed that it was a carcinoma. The patient was then placed in the supine position to undergo mediastinoscopy with biopsy of the lesion, with the gantry set to fluoroscopic mode. Examination of a frozen section biopsy specimen confirmed an epidermoid carcinoma in the infracarinal lymph node. In view of the diagnosis of (stage N2) lung cancer, we proceeded to

Correspondence to:

Ricardo M. Terra. Instituto do Coração, HC-FMUSP, Avenida Dr. Enéas de Carvalho Aguiar, 33, Bloco II, Sala 9, Cerqueira César, CEP 05403-000, São Paulo, SP, Brasil.

Tel.: 5511 2661-5248 or 5511 2661-5000. E-mail: rmterra@uol.com.br

Financial support: None. 
insert a catheter for chemotherapy, again with the gantry set to fluoroscopic mode.

The use of an HOR in the case described here allowed all procedures to be performed sequentially, in the same setting, without need for transportation. In addition, we would have the flexibility of adding pulmonary lobectomy if the pulmonary mass was positive for cancer and did not extend into the mediastinum. We were planning to use the gantry set to tomographic mode during mediastinoscopy in order to assist the intraoperative localization of the mediastinal lesion. However, the vertical size of the video mediastinoscope system precluded free rotation of the gantry, which is required to generate the tomographic image, and it was possible to use only fluoroscopy, which dispenses with complete rotation of the gantry.

\section{Case 2}

A 62-year-old man presented with a history of metastatic rectal cancer to the liver at diagnosis. He was treated with neoadjuvant chemotherapy, followed by resection of a liver lesion and ablation of another liver lesion. Subsequently, the patient received chemotherapy and radiotherapy of the rectal lesion, followed by resection of the rectal tumor. Finally, he received adjuvant chemotherapy totaling 12 cycles, including the initial treatment. Eighteen months after diagnosis, the patient developed two pulmonary lesions in the left upper lobe, suggestive of metastases. Those lesions remained stable for more than 6 months. After that period, they showed slight growth and a new finding was observed in the right lower lobe. The patient was considered for pulmonary metastasectomy; however, he wanted the surgical procedure to be performed minimally invasively. The lesion on the right side was deep; therefore, it would be impossible to resect it by video-assisted thoracoscopic surgery without lobectomy. After a multidisciplinary meeting, we opted for performing right-sided radiofrequency ablation and left-sided video-assisted thoracoscopic surgery. The procedures were performed in an HOR, with the patient undergoing general anesthesia and elective endotracheal intubation. The patient was initially placed in the left lateral decubitus position for right-sided radiofrequency ablation using a radiofrequency ablation probe (ACT2030; Covidien, Boulder, CO, USA), with the gantry (Artis Zeego) set to tomographic mode, and the procedure was uneventful. Subsequently, the patient was placed in the right lateral decubitus position and underwent left-sided video-assisted thoracoscopic surgery. One of the nodules was identified on the lung surface; the other, because of its greater depth, was identified intraoperatively with the gantry set to tomographic mode, a technique that some authors have termed image-guided video-assisted thoracoscopic surgery $^{(6)}$ (iVATS, Figure 1). Pathological examination of the two resected lesions confirmed that they were metastatic adenocarcinomas of colorectal origin, both of which had free margins.
The availability of an HOR not only allowed the scheduling of the minimally invasive procedures in the same surgical session, but also made it possible to locate the slightly deeper lesion easily and safely, through the use of iVATS.

\section{Case 3}

A 57-year-old female smoker (with a smoking history of 40 pack-years) presented with a coronary artery CT finding of a $1.7-\mathrm{cm}$ semisolid nodule with a 3-mm solid component. Three months later, the solid component had increased to $5 \mathrm{~mm}$ (Figure 2A). The lesion was located close to the transition between the upper segment of the left lower lobe and the basal pyramid. Because the lesion was deep and showed a predominant ground-glass pattern, difficulties were to be expected in locating it intraoperatively, as well as in establishing resection margins. Therefore, we planned to inject the nodule with lipiodol (Guerbet, Villepinte, France), making it radiopaque, which would allow its radiological identification during resection. ${ }^{(7)}$ In the HOR, the patient underwent general anesthesia with elective endotracheal intubation and was placed in the right lateral decubitus position, already in the surgical position. An interventional radiologist identified the nodule using a CT scanner and injected it with $0.3 \mathrm{~mL}$ of lipiodol, which resulted in excellent fluoroscopic and tomographic views of the nodule (Figure 2B). Subsequently, a guidewire was inserted to assist in guidance on the lung surface anatomy. A muscle-sparing thoracotomy at the 5th intercostal space was then performed, after which anatomic segmentectomy of the upper segment of the left lower lobe, including part of the basal segments, was performed, as well as hilar lymphadenectomy. The intersegmental resection line was developed through stapling, being guided by the radiopaque image produced by the CT scanner and by fluoroscopy (Figure 2C), in order to achieve adequate margins for the whole nodule (Figures 2D and 2E). Surgery and anesthesia were uneventful, and the patient was extubated at the end of the procedure. Pathological examination revealed a lepidic-predominant adenocarcinoma with a minimum margin of $15 \mathrm{~mm}$.

The use of the HOR imaging tools, as well as lipiodol injection of the nodule, was effective in guiding the localization not only of the nodule, but also of the resection margin. The addition of lipiodol was effective, allowing satisfactory identification of the nodule, and seemed more advantageous than using a guidewire alone, given that the wire often moves during lung mobilization and does not indicate to the surgeon the exact position of the lesion.

\section{DISCUSSION}

In this initial experience with an HOR, we obtained satisfactory results; however, it is important to emphasize that there is a learning curve with the use of its tools. First, the use of the imaging equipment 


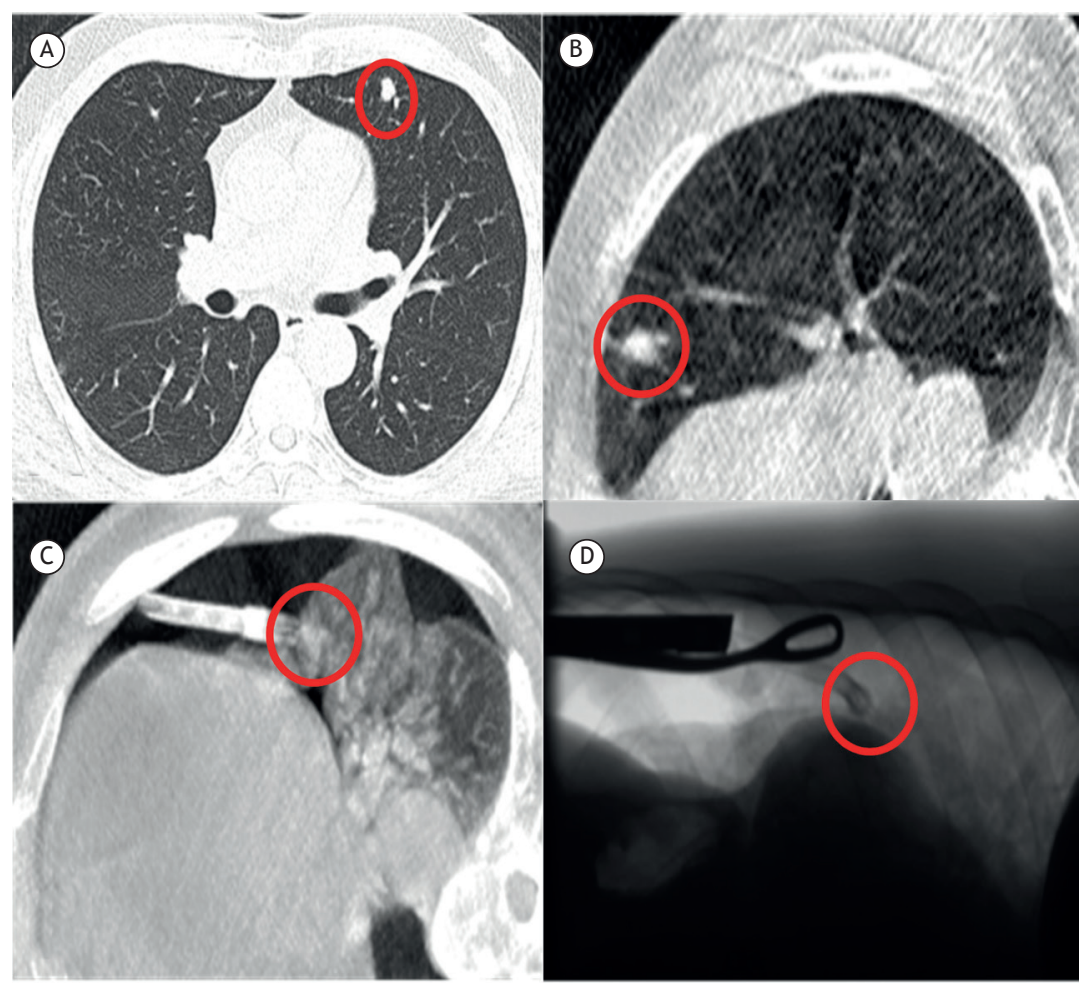

Figure 1. Case 2: In $A$, a preoperative $C T$ scan. In $B$, an intraoperative $C T$ scan identifying a nodule in the inflated lung. In $\mathrm{C}$, an intraoperative CT scan identifying a nodule in the collapsed lung. In D, intraoperative fluoroscopy with a probe in place, ensuring the achievement of clear margins for the previously localized nodule.
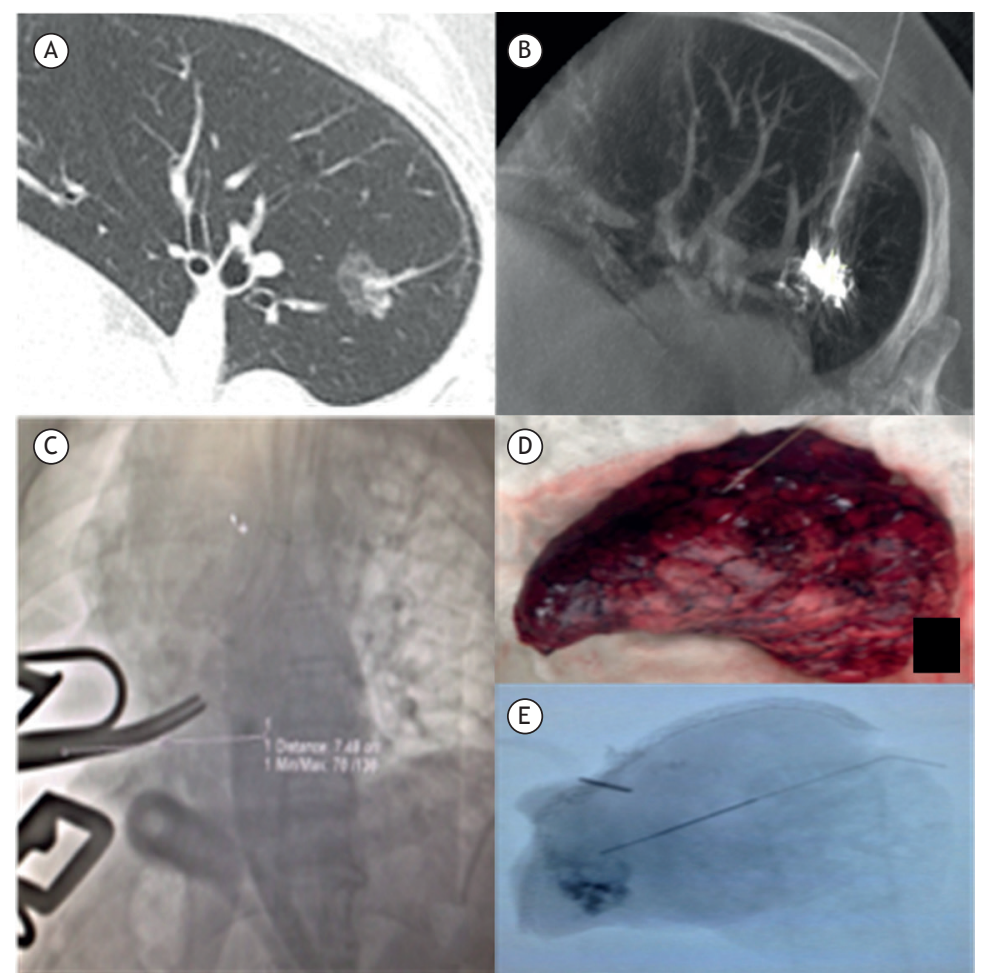

Figure 2. Case 3. In A, a CT scan showing a semisolid nodule. In B, an intraoperative CT scan demonstrating lipiodol uptake in the nodule. In $\mathrm{C}$, intraoperative fluoroscopy with a probe ensuring the achievement of clear margins for the nodule with lipiodol uptake. In $D$, a photograph of the surgical specimen taken from the upper segment of the lower lobe. In $\mathrm{E}$, an X-ray of the surgical specimen, identifying the nodule with lipiodol uptake and adequate margins. 
poses a challenge to radiologists, given that the interface is a little different from that usually found in interventional radiology services. Second, patient positioning, as well as equipment and staff positioning, requires special care, ${ }^{(8)}$ since gantry rotation should be taken into account. The patient's arms should be placed at his/her sides so as not to exceed the width of the operating table; and the use of cushions/pads is essential, because the operating table is flat and very limited in terms of mobilization. Finally, as in any surgical procedure, a larger number of cases would increase the consistency and efficiency of the procedures, consequently reducing room time, which was relatively long in the examples described here.

The decisive factor for the success of the cases reported here was certainly the integration of the surgical, anesthetic, and radiological teams. Only after considerable discussion regarding the needs and contributions of each team were we able to proceed to perform the procedures. The key issue for debate is certainly positioning, because the initially most suitable positioning for surgery was not appropriate for radiology. The first change in positioning was a cause of concern for the anesthetic team, who was vigilant for injuries. Further discussions were held at each adaptation, until positioning was approved by all.

In conclusion, in the three cases reported here, the use of an HOR was feasible and seems to have facilitated performing the procedures. Most importantly, through these cases, we could envision potential applications for an HOR in thoracic surgery, in particular, the possibility of performing diagnostic and therapeutic procedures in the same setting and the possibility of resecting very small lesions accurately. In the current context of increased use of minimally invasive sublobar resections and of an increase in early diagnosis of lung cancer by screening programs, the tools offered by an HOR are promising and merit scientific research.

\section{REFERENCES}

1. Ng I. Integrated intra-operative room design. Acta Neurochir Suppl. 2011;109:199-205. http://dx.doi.org/10.1007/978-3-211-99651-5_31

2. Scolozzi $P$, Schouman T. Interventional multidimodal hybrid unit: from pre-operative planning to immediate post-operative control [Article in French]. Rev Stomatol Chir Maxillofac. 2012;113(2):11523. http://dx.doi.org/10.1016/j.stomax.2012.01.009

3. Yao C, Liu Y, Yao J, Zhuang D, Wu J, Qin Z, et al. Augment lowfield intra-operative MRI with preoperative MRI using a hybrid nonrigid registration method. Comput Methods Programs Biomed. 2014;117(2):114-24. http://dx.doi.org/10.1016/j.cmpb.2014.07.013

4. Harskamp RE, Puskas JD, Tijssen JG, Walker PF, Liberman HA, Lopes RD, et al. Comparison of hybrid coronary revascularization versus coronary artery bypass grafting in patients $\geq 65$ years with multivessel coronary artery disease. Am J Cardiol. 2014;114(2):224
9. http://dx.doi.org/10.1016/j.amjcard.2014.04.028

5. Varu VN, Greenberg JI, Lee JT. Improved efficiency and safety for EVAR with utilization of a hybrid room. Eur J Vasc Endovasc Surg. 2013;46(6):675-9. http://dx.doi.org/10.1016/j.ejvs.2013.09.023

6. Finley RJ, Clifton J, Mayo J. Image-guided video-assisted thoracoscopic resection of small peripheral lung nodules. Adv Surg. 2005;39:263-84. http://dx.doi.org/10.1016/j.yasu.2005.03.001

7. Kim YD, Jeong YJ, I H, Cho JS, Lee JW, Kim HJ, et al. Localization of pulmonary nodules with lipiodol prior to thoracoscopic surgery. Acta Radiol. 2011;52(1):64-9. http://dx.doi.org/10.1258/ar.2010.100307

8. Hemingway M, Kilfoyle M. Safety planning for intraoperative magnetic resonance imaging. AORN J. 2013;98(5):508-24. http:// dx.doi.org/10.1016/j.aorn.2013.09.002 